\section{Potencia aeróbica máxima en el obeso: Valores de referencia}

\author{
JORGE CANCINO-LÓPEZ ${ }^{1, \mathrm{c}}$, MARIELA OLIVARES-GÁLVEZ ${ }^{2, \mathrm{a}}$, \\ CRISTÓBAL MAIZ-HOHLBERG ${ }^{3,}$ JOHANA SOTO-SÁNCHEZ,c, \\ ANA PALACIO-AGÜERO ${ }^{5,6, b}$, JOHANNA PINO-ZÚÑIGA ${ }^{2, a}$
}

\section{Maximum aerobic power output in overweight and obese individuals}

Background: The evaluation of physical fitness is important to determine workloads and to assess the effectiveness of exercise interventions in obese people. Aim: To determine the maximum aerobic power output (MAPO) in overweight and obese individuals and to establish reference parameters for the Chilean population. Material and Methods: One hundred five men and 218 women performed a cycle ergometer test up to $85 \%$ of their maximum heart rate. MAPO was determined by linear extrapolation of the theoretical maximum heart rate. Results: Among men, MAPO values were $234.9 \pm 48.4 w$ for body mass index (BMI) values between 30 and $34.9 \mathrm{~kg} / \mathrm{m}^{2}, 235.1 \pm 69.7 w$ for BMI between 35 and $39.9 \mathrm{~kg} / \mathrm{m}^{2}, 270.2 \pm 86.5 \mathrm{w}$ for BMI over $40 \mathrm{~kg} / \mathrm{m}^{2}$. MAPO relative values were $2.27 \pm 0.5,2.16 \pm 0.6$ and $1.96 \pm 0.8 \mathrm{w} / \mathrm{kg}$, respectively. Among women, MAPO was $172.6 \pm 36.1 \mathrm{w}$ for a $B M I<30 \mathrm{~kg} / \mathrm{m}^{2}, 169.2 \pm 39.4 \mathrm{w}$ for BMI between 30 and $34.9 \mathrm{~kg} / \mathrm{m}^{2}, 179.5 \pm 48.8 w$ f179.5 $\pm 48.8 w$ for BMI between 35 and $39.9 \mathrm{~kg} / \mathrm{m}^{2}$ and $202.3 \pm 57.3 w$ for BMI of $40 \mathrm{~kg} / \mathrm{m}^{2}$ or over. The relative values were $2.33 \pm 0.5 ; 2.02 \pm 0.5 ; 1.91 \pm 0.5$ and $1.81 \pm 0.5 \mathrm{w} / \mathrm{kg}(p<0.05)$. Conclusions: $M A P O$ estimation with a cycle ergometer test is well tolerated in overweight and obese individuals. MAPO decreased along with BMI increments in women. No association between BMI and MAPO in men was observed.

(Rev Med Chile 2019; 147: 289-295)

Key words: Body Mass Index; Obesity.

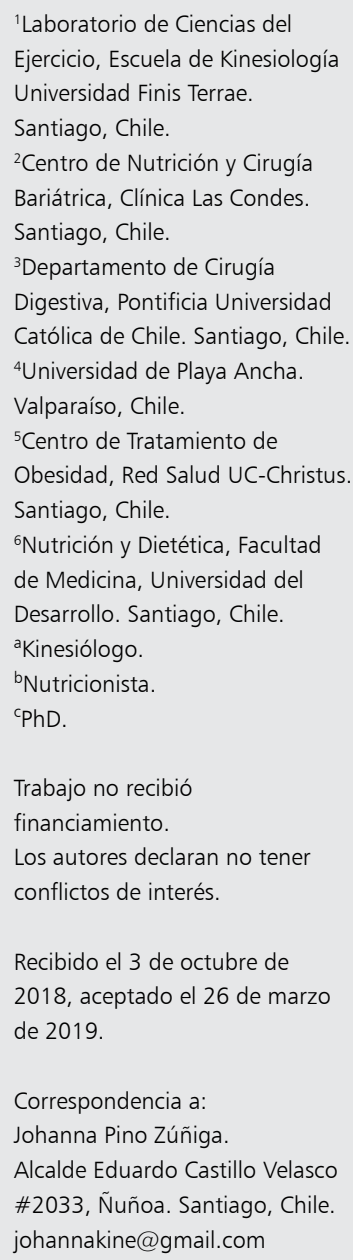

L

a obesidad se caracteriza por un incremento excesivo de la grasa corporal, originado por un balance energético positivo mantenido en el tiempo, pudiendo ser originada ya sea por un exceso de ingesta y/o un déficit en el gasto energético ${ }^{1,2}$. La obesidad constituye un factor de riesgo frente a numerosas enfermedades, tales como diabetes mellitus 2, hipertensión arterial, dislipidemia, entre otras ${ }^{3}$.

Por otra parte, la condición física se relaciona con la capacidad de generar trabajo, la independencia funcional y la calidad de vida en personas de diferente edad y condiciones patológicas ${ }^{4-10}$. Se ha relacionado la menor condición física en obesos con un mayor riesgo cardiovascular ${ }^{11}$. Por lo tanto, la evaluación de la misma, resulta indispensable en la práctica clínica, tanto para conocer el estado actual del paciente como para planificar objetivos y evaluar progresos en el tiempo. Diversos estudios demuestran que individuos con obesidad presentan una disminución de su capacidad física al ser comparados con individuos normopesos ${ }^{12-14}$. Se ha sugerido que la disminución de la capacidad física se debe al aumento de la demanda metabólica de los tejidos extramusculares y que, por lo tanto, al bajar de peso, la capacidad física mejora por sí sola, independientemente del entrenamiento específico de ésta ${ }^{15-17}$. 
El consumo máximo de oxígeno $\left(\mathrm{VO} 2_{\text {máx }}\right)$ corresponde a la máxima cantidad de oxígeno que el organismo es capaz de absorber, transportar y utilizar por unidad de tiempo ${ }^{18}$. La medición directa del mismo, representa el “estándar de oro" para la valoración de la capacidad funcional máxima de las personas, y su asociación con la potencia aeróbica máxima (PAM), cuando se mide en el ciocloergómetro o mediante velocidad aeróbica máxima (VAM) alcanzada en el treadmill, permite hacer prescripciones de ejercicio y posibilita el control de la sesión de entrenamiento al existir una relación lineal entre el comportamiento del consumo de oxígeno durante la prueba incremental y la frecuencia cardiaca ${ }^{19}$. Sin embargo, se debe tener en consideración que el costo del equipamiento y los requerimientos técnicos de la operación, hacen que no sea una prueba de valoración habitual en población no deportista. Para estas situaciones, la valoración indirecta de la capacidad funcional a través de pruebas de valoración submáximas resulta más adecuada.

Una forma de evaluación lo representan test indirectos que se basan en la relación lineal entre la frecuencia cardiaca (FC) y la potencia o carga de trabajo, permitiendo la extrapolación de potencias a niveles de frecuencias cardiacas determinadas, ya sea absolutas (130, 150, 170 pulsaciones) o relativas al porcentaje de la frecuencia cardiaca máxima teórica $(60,70,85 \%)^{12,20}$.

La evaluación de la condición física tradicionalmente se realiza en treadmill o cicloergómetro. El beneficio de utilizar un test en cicloergómetro a diferencia de utilizar treadmill en pacientes con obesidad se relaciona con que no deben soportary trasladar el peso de su cuerpo, ya que estos presentan una marcha que biomecánicamente es ineficiente, lo que implica que en los test de caminata, el menor rendimiento reportado podría deberse no a una capacidad fisiológica disminuida, sino a factores como los mencionados anteriormente ${ }^{17}$.

La potencia mecánica, medida en watts, durante un test en cicloergómetro ha sido valorada tanto en condiciones de esfuerzo máximo como submáximo. Al respecto, la potencia mecánica que una persona puede realizar a una frecuencia cardíaca de 170 latidos por minuto $\left(\mathrm{P}_{170}\right)$, se ha utilizado para evaluar la condición física de sujetos sanos que se someten a un programa de entrenamiento, adolescentes con obesidad y síndrome metabólico, deportistas recreacionales y en diver- sos estudios para relacionar condición física con riesgo cardiovascular ${ }^{12,21-23}$. Sin embargo, presenta una limitación para la población adulta, debido a que la frecuencia cardiaca máxima se ve reducida con la edad. Así, una frecuencia de 170 latidos por minutos podría, para una persona de 50 años corresponder a su frecuencia cardiaca máxima.

No existen datos publicados en Chile sobre la Potencia aeróbica máxima (PAM), obtenida a partir de test indirectos submáximos, del paciente obeso con una muestra lo suficientemente amplia como para obtener valores de referencia. Por lo anterior, el objetivo de este estudio fue determinar la PAM en cicloergómetro a través de un test indirecto y submáximo de extrapolación lineal de la frecuencia cardiaca en pacientes obesos y valorar si el nivel de obesidad es un factor limitante de dicho rendimiento.

\section{Materiales y Métodos}

Se realizó un estudio de cohorte retrospectivo que consistió en recopilar la información obtenida en la prueba de PAM de 319 pacientes adultos con exceso de peso, obesos y sedentarios, que iniciaron tratamiento en en un centro de manejo de la obesidad de la Región Metropolitana. Fueron excluidos aquellos pacientes que utilizaban medicamentos que alteraran su frecuencia cardíaca (betabloqueadores), y aquellos que por alguna razón no pudieran realizar ejercicio en cicloergómetro. El procedimiento de recolección de datos y confidencialidad de estos se ajustó a las directrices que plantea el Comité de Ética de la Pontificia Universidad Católica de Chile (No de proyecto 14240, año 2014).

\section{Valoración de la PAM}

La prueba consistió en un test incremental en cicloergómetro (Lifefitness $9500 \mathrm{HR}^{\circledast}$ ). La carga inicial para mujeres fue de 50 watts y para hombres de 75 watts. Los pacientes debían mantener una cadencia de pedaleo de 50 revoluciones por minuto (RPM) y cada 2 min se fue aumentando la carga en 25 watts. El test se detuvo cuando llegaban a una frecuencia cardiaca equivalente al $85 \%$ de su frecuencia cardiaca máxima teórica o hasta que no pudieran mantener la cadencia de pedaleo. La frecuencia cardiaca se registró con una banda pectoral y transmitida a un receptor de muñeca (Polar, Modelo FT1, Finlandia). 
El cálculo de la PAM fue realizado por extrapolación lineal de la frecuencia cardiaca. Se consideraron las dos últimas cargas de ejercicio en el cicloergómetro y las frecuencias cardiacas correspondientes a dichas cargas. Para la determinación de la frecuencia cardiaca máxima teórica se utilizó la fórmula de 220-edad. Dicha elección se realizó siguiendo los resultados de Storer et al. $1990^{24}$, quien reportó una diferencia entre la frecuencia cardiaca máxima medida y la estimada de tan solo 2,8 latidos/min para un test en cicloergómetro.

Para el cálculo de la PAM se utilizó la ecuación:

$\mathrm{PAM}=\mathrm{C} 1+(\mathrm{C} 2-\mathrm{C} 1) /(\mathrm{FC} 2-\mathrm{FC} 1) *\left(\mathrm{FC}_{\text {máx }}-\mathrm{F} 1\right)$

Donde:

PAM = Potencia aeróbica máxima.

$\mathrm{C} 1$ = Carga anterior a la última carga de trabajo realizada en el cicloergómetro.

C2 = Última carga de trabajo realizada en el cicloergómetro.

FC1 = Frecuencia cardiaca alcanzada al final del segundo minuto de la carga $\mathrm{C} 1$.

FC2 = Frecuencia cardiaca alcanzada en el segundo minuto de la carga $\mathrm{C} 2$.

$\mathrm{FC}_{\text {máx }}=$ Frecuencia cardiaca máxima según la fórmula 220-edad.

Una vez obtenida la PAM, el valor relativo se determinó dividiendo el valor absoluto en el peso corporal de cada participante. El valor absoluto de PAM se transformó además a valores de METs según ecuaciones metabólicas del Colegio Americano de Medicna del Deporte ${ }^{25}$.
Al finalizar el test se realizaron 5 min de trabajo con la carga de inicio a modo de recuperación activa.

La normalidad en la distribución de los datos fue evaluada mediante el test de Shapiro Wilk. Para la comparación del grado de obesidad (definido por IMC) entre los diferentes grupos, se utilizó la prueba de ANOVA one way y el test post hoc de Bonferroni cuando los datos distribuyeron normal, en caso contrario se utilizó el test de Kruskall Wallis con el post hoc de Dunn.

Las diferencias fueron consideradas estadísticamente significativas toda vez que $\mathrm{p}<0,05$. Los análisis fueron realizados con el programa estadístico STATA 12,1 (Stata Corp, College Station, TX).

\section{Resultados}

Las características de los pacientes se detallan en las Tablas 1 y 2 , para hombres y mujeres respectivamente.

La PAM para hombres (Tabla 1) según IMC, fue: IMC $30-34,9 \mathrm{~kg} / \mathrm{m}^{2}: 234,9 \pm 48,4 \mathrm{w}$; IMC $35-39,9 \mathrm{~kg} / \mathrm{m}^{2}: 235,1 \pm 69,7 \mathrm{w} ; \mathrm{IMC} \geq 40 \mathrm{~kg} / \mathrm{m}^{2}$ : $270,2 \pm 86,5 \mathrm{w}$. El valor relativo fue $2,27 \pm 0,5$; $2,16 \pm 0,6$ y $1,96 \pm 0,8 \mathrm{w} / \mathrm{kg}$, respectivamente. El valor expresado en METs fue 9,0 $\pm 1,2 ; 8,97 \pm 2,46$ y $8,17 \pm 1,87$ respectivamente. No se observaron diferencias significativas en los valores de la PAM, PAM relativa y METs entre los 3 grupos de IMC en hombres. En las mujeres la PAM (Tabla 2) según IMC, fue: IMC $<30 \mathrm{~kg} / \mathrm{m}^{2}: 172,6 \pm 36,1 \mathrm{w} ; \mathrm{IMC}$ $30-34,9 \mathrm{~kg} / \mathrm{m}^{2}: 169,2 \pm 39,4 \mathrm{w}$; IMC $35-39,9 \mathrm{~kg} /$

Tabla 1. Parámetros en hombres según IMC (valores expresados en media y DE)

\begin{tabular}{|c|c|c|c|c|}
\hline & $\begin{array}{c}30-34,9\left(\mathrm{~kg} / \mathrm{m}^{2}\right) \\
(\mathrm{n}=39)\end{array}$ & $\begin{array}{c}35-39,9\left(\mathrm{~kg} / \mathrm{m}^{2}\right) \\
(\mathrm{n}=42)\end{array}$ & $\begin{aligned} \geq & 40\left(\mathrm{~kg} / \mathrm{m}^{2}\right) \\
& (\mathrm{n}=24)\end{aligned}$ & Valor $\mathbf{p}$ \\
\hline Edad (años) & $34,5 \pm 11,14$ & $34,02 \pm 10,8$ & $31,5 \pm 8,1$ & 0,61 \\
\hline IMC $\left(\mathrm{kg} / \mathrm{m}^{2}\right)$ & $32,92 \pm 1,3$ & $36,59 \pm 1,2$ & $43,27 \pm 4,4$ & $<0,0001$ \\
\hline PAM (w) & $234,9 \pm 48,4$ & $235,1 \pm 69,7$ & $270,2 \pm 86,5$ & $0,083^{+}$ \\
\hline PAM (w/kg) & $2,27 \pm 0,5$ & $2,16 \pm 0,6$ & $1,96 \pm 0,8$ & $0,1639^{+}$ \\
\hline METS & $9,0 \pm 1,2$ & $8,97 \pm 2,46$ & $8,17 \pm 1,87$ & $0,206^{*}$ \\
\hline$P_{170}(w)$ & $194,6 \pm 45,6$ & $192,1 \pm 48,8$ & $224,98 \pm 81,1$ & $0,060^{*}$ \\
\hline$P_{170}(w / k g)$ & $1,88 \pm 0,36$ & $1,76 \pm 0,46$ & $1,70 \pm 0,62$ & $0,276^{+}$ \\
\hline$\% F C_{\text {máx }}$ test & $84,19 \pm 7,8$ & $86,25 \pm 6,8$ & $83,1 \pm 5,3$ & $0,176^{*}$ \\
\hline
\end{tabular}

${ }^{*}$ Anova. ${ }^{+}$Kruskal Wallis. 
Tabla 2. Parámetros en mujeres según IMC (valores expresados en media y DE)

\begin{tabular}{|c|c|c|c|c|c|}
\hline IMC (kg/m²) & $\begin{array}{c}<30 \\
(n=46)\end{array}$ & $\begin{array}{c}30-34,9 \\
(n=110)\end{array}$ & $\begin{array}{l}35-39,9 \\
(n=43)\end{array}$ & $\begin{array}{c}\geq 40 \\
(n=19)\end{array}$ & Valor p \\
\hline Edad (años) & $30,8 \pm 8,5$ & $33,33 \pm 9,4$ & $31,4 \pm 7,2$ & $29,7 \pm 10,6$ & 0,58 \\
\hline IMC (kg/m²) & $27,9 \pm 1,5$ & $32,5 \pm 1,7$ & $37,2 \pm 1,9$ & $43,4 \pm 4,1$ & $<0,0001$ \\
\hline PAM (w) & $172,6 \pm 36,1^{1}$ & $169,2 \pm 39,4$ & $179,5 \pm 48,8$ & $202,3 \pm 57,3$ & $<0,001^{+}$ \\
\hline PAM (w/kg) & $2,33 \pm 0,5^{1}$ & $2,02 \pm 0,5$ & $1,91 \pm 0,5$ & $1,81 \pm 0,5$ & $<0,016^{+}$ \\
\hline METs & $9,08 \pm 1,54^{1}$ & $8,2 \pm 1,24$ & $7,7 \pm 1,66$ & $7,2 \pm 1,76$ & $<0,0001$ \\
\hline$P_{170}(w)$ & $143,9 \pm 34$ & $137,6 \pm 26$ & $142,9 \pm 34$ & $156,8 \pm 41$ & $0,093^{*}$ \\
\hline$P_{170}(w / k g)$ & $1,89 \pm 0,40$ & $1,66 \pm 0,35$ & $1,52 \pm 0,38$ & $1,39 \pm 0,30^{1}$ & $<0,0001$ * \\
\hline$\%$ FC máx test & $84,2 \pm 6,1$ & $86,3 \pm 5,9$ & $86,6 \pm 4,74$ & $84,3 \pm 5,5$ & $0,085^{*}$ \\
\hline
\end{tabular}

*Anova; ${ }^{*}$ Kruskal Wallis; ${ }^{1}$ Este es el valor diferente de la serie.

Tabla 3. Valores percentiles de la PAM absoluta (w); relativa (w/kg) y METs en hombres y mujeres

\begin{tabular}{|c|c|c|c|c|c|}
\hline \multicolumn{2}{|c|}{ IMC (Kg/m²) } & \multirow{2}{*}{$\begin{array}{l}\quad \begin{array}{l}<\mathbf{3 0} \\
(\mathbf{n}=\mathbf{4 6})\end{array} \\
\text { No } \\
147,6 \quad(1,94) \\
\text { No } \\
\quad 7,9\end{array}$} & $\begin{array}{c}\mathbf{3 0 - 3 4 , 9} \\
(\mathbf{n}=\mathbf{1 1 0 ~} \mathbf{M} ; \mathbf{3 9} \mathbf{H}) \\
200 \quad(1,95)\end{array}$ & \multirow{2}{*}{ 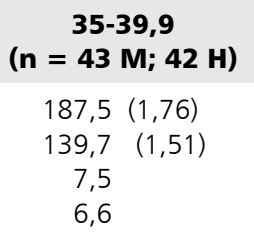 } & \multirow{2}{*}{$\begin{array}{c}\mathbf{\geq} \mathbf{4 0} \\
(\mathbf{n}=\mathbf{1 9} \mathbf{~ M ;} \mathbf{2 4} \mathbf{H}) \\
200 \quad(1,53) \\
158,7 \quad(1,49) \\
6,8 \\
6,6\end{array}$} \\
\hline $\begin{array}{l}\text { P25 } \\
\text { w (w/kg) } \\
\text { METs }\end{array}$ & $\begin{array}{l}\text { Hombres } \\
\text { Mujeres } \\
\text { Hombres } \\
\text { Mujeres }\end{array}$ & & $\begin{array}{rr}200 & (1,95) \\
144,4 & (1,70) \\
8,2 & \\
7,5 & \end{array}$ & & \\
\hline $\begin{array}{l}\text { P50 } \\
\text { w (w/kg) } \\
\text { METs }\end{array}$ & $\begin{array}{l}\text { Hombres } \\
\text { Mujeres } \\
\text { Hombres } \\
\text { Mujeres }\end{array}$ & $\begin{array}{l}\text { No } \\
165,1 \quad(2,18) \\
\text { No } \\
\quad 8,8\end{array}$ & $\begin{array}{rr}229,2 & (2,17) \\
160,2 & (1,94) \\
8,7 & \\
8,1 & \end{array}$ & $\begin{aligned} 219,9 & (1,98) \\
166,9 & (1,73) \\
8,2 & \\
7,3 & \end{aligned}$ & $\begin{array}{rr}259,7 & (1,94) \\
186,4 & (1,84) \\
8,1 & \\
7,5 & \end{array}$ \\
\hline $\begin{array}{l}\text { P75 } \\
\text { w (w/kg) } \\
\text { METs }\end{array}$ & $\begin{array}{l}\text { Hombres } \\
\text { Mujeres } \\
\text { Hombres } \\
\text { Mujeres }\end{array}$ & $\begin{array}{l}\text { No } \\
191,1 \quad(2,68) \\
\text { No } \\
10,1\end{array}$ & $\begin{aligned} 273,5 & (2,62) \\
180,9 & (2,20) \\
10,1 & \\
8,9 & \end{aligned}$ & $\begin{aligned} 246,9 & (2,39) \\
210,2 & (2,17) \\
9,7 & \\
8,5 & \end{aligned}$ & $\begin{array}{rr}304,2 & (2,22) \\
227,3 & (2,03) \\
8,9 & \\
8,3 & \end{array}$ \\
\hline
\end{tabular}

$\mathrm{m}^{2}: 179,5 \pm 48,8 \mathrm{w} ; \mathrm{IMC} \geq 40 \mathrm{~kg} / \mathrm{m}^{2}: 202,3 \pm 57,3$ $\mathrm{w}$, sin diferencias significativas. El valor relativo fue $2,33 \pm 0,5 ; 2,02 \pm 0,5 ; 1,91 \pm 0,5$ y $1,81 \pm 0,5$ $\mathrm{w} / \mathrm{kg}$, observándose diferencias significativas entre ellas $(\mathrm{p}<0,05)$. El valor expresado en METs fue de $9,08 \pm 1,54 ; 8,2 \pm 1,24 ; 7,7 \pm 1,66$ y $7,2 \pm 1,76$, existiendo diferencias significativas entre IMC $<30 \mathrm{~kg} / \mathrm{m}^{2}$ con el resto de los grupos y entre IMC $30-34,9 \mathrm{~kg} / \mathrm{m}^{2}$ con IMC $35-39,9 \mathrm{~kg} / \mathrm{m}^{2}$. El percentil 50 (p50) para hombres fue según: IMC 30-34,9 $\mathrm{kg} / \mathrm{m}^{2}: 229,2(2,17)$; IMC $35-39,9 \mathrm{~kg} / \mathrm{m}^{2}: 219,9$ $(1,98) ; \mathrm{IMC} \geq 40 \mathrm{~kg} / \mathrm{m}^{2}: 259,7$ (1,94) expresados en w y $(\mathrm{w} / \mathrm{kg})$ respectivamente. El valor expresado en METs fue de 8,7; 8,2 y 8,1 respectivamente. En mujeres fue: IMC $<30 \mathrm{~kg} / \mathrm{m}^{2}: 165(2,18)$; IMC 30$34,9 \mathrm{~kg} / \mathrm{m}^{2}: 160,2$ (1,94); IMC 35-39,9 kg/m²: 166,9
$(1,73) ; \mathrm{IMC} \geq 40 \mathrm{~kg} / \mathrm{m}^{2}: 186,4(1,84)$ expresados en w y $(\mathrm{w} / \mathrm{kg})$ respectivamente. El valor expresado en METs fue de 8,8; 8,1: 7,3 y 7,5 (Tabla 3).

\section{Discusión}

Se ha descrito la potencia aeróbica máxima del paciente con obesidad a través de un método indirecto y submáximo que permite una fácil replicación sin la necesidad de equipos de alto costo. Hasta aquí, se pensaba que estos pacientes presentaban una capacidad funcional disminuida a consecuencia de su enfermedad. Sin embargo, al analizar su potencia aeróbica máxima, se aprecia que en términos absolutos no es menor, sino en 
ocasiones incluso mayor a la descrita en poblaciones no obesas ${ }^{26,27,12}$. Dado que la evaluación se realiza durante una actividad que no requiere transportar el peso corporal, esta variable deja de ser una limitante desde el punto de vista fisiológico. La generación de potencia sobre un cicloergómetro depende de la apropiada transformación de energía química producida por la musculatura en energía mecánica que luego se traduce a los pedales. Para esto, es necesario que los sistemas respiratorio, cardiovascular y muscular actúen de manera coordinada para transformar el oxígeno y los sustratos energéticos en adenosintrifosfato (ATP) a una velocidad tal que permita mantener una potencia mecánica adecuada ${ }^{28}$.

Si bien, la capacidad para realizar trabajo mientras se transporta el peso corporal, está disminuida en el paciente con obesidad ${ }^{29-32}$, esto no parece ser así cuando dicho paciente es enfrentado a actividades en las cuales no debe transportar su peso como es el caso del cicloergómetro ${ }^{33}$. Cuando se realiza un ejercicio con las extremidades inferiores, la actividad muscular provocará una hiperemia activa vinculada a condiciones generadas tanto en el propio músculo, como en las células endoteliales $^{34,35}$. La potencia mecánica generada en el cicloergómetro dependerá de la masa muscular activa y de la capacidad de esta para transformar el oxígeno en energía contráctili ${ }^{28}$. Es requisito para ello, que exista un incremento en el flujo sanguíneo a las extremidades ejercitadas. Se sabe que la volemia está aumentada en los obesos, por lo que no existiría una limitante en cuanto a la capacidad de transporte de oxígeno hacia la musculatura mientras la función cardiaca no esté comprometida $^{13}$. Es por esta misma característica, que de no estar limitada la capacidad de la bomba cardiaca y de ser efectiva la capacidad vasodilatadora muscular en oponerse a la actividad simpática, la que se sabe que es mayor en los obesos ${ }^{36}$, debería existir un adecuado flujo sanguíneo muscular para la producción de energía. Otro elemento que podría estar jugando a favor de la capacidad funcional en el obeso cuando es analizado su desempeño en el cicloergómetro es el peso de la extremidad inferior, ya que para producir el desplazamiento de los pedales, se requiere de una fuerza vertical descendente, la cual es parte del movimiento circular que se realiza mientras se pedalea sobre una bicicleta ${ }^{28}$. También podría considerarse que a causa de transportar un mayor peso corporal en actividades de la vida diaria, la sobrecarga ejercida sobre la extremidad inferior podría significar un mayor estímulo de entrenamiento para dicha musculatura y por ende desarrollar un mayor nivel de fuerza, lo que ha sido reportado tanto en adolescentes como en adultos obesos ${ }^{37,38}$. Esta masa y fuerza sería directamente traspasada a los pedales cuando el paciente con obesidad realiza ejercicio sobre el cicloergómetro permitiendo, si el sistema cardiovascular no lo limita, el desarrollo de una potencia mecánica adecuada durante esta actividad.

Debido a que la potencia determinada para los pacientes con obesidad en este trabajo, no sería menor a la reportada en otras poblaciones normopeso en términos absolutos ${ }^{26,27,12}$ y que a la hora de desarrollar esfuerzo físico en actividades que no requieren transportar todo el peso corporal, la potencia relativa pierde importancia, es que se hace necesario discutir sobre la forma en que esta población puede y debe ser abordada en cuanto a la realización de ejercicio físico. Es reconocido que la prescripción de ejercicio debe ser un elemento fundamental para este tipo de pacientes ${ }^{39-44}$ y que también la adherencia a programas de actividad física mejora en la medida que estos son mejor tolerados por las personas ${ }^{45}$. El ejercicio físico es fundamental para el paciente con obesidad y en especial para aquellos sometidos a cirugía bariátrica. Si bien es sabido que dichos procedimientos generan una pérdida de masa muscular importante, esta puede ser aminorada mediante la realización de actividad física programada ${ }^{46}$. La evaluación y la mejora de la capacidad funcional del paciente con obesidad es además importante considerando que una mayor capacidad funcional podría ser beneficiosa al momento de la cirugía bariátrica, favoreciendo una menor tasa de complicaciones postoperatorias y una más rápida recuperación ${ }^{47}$.

El grado de obesidad no demostró ser un elemento que afecte la potencia aeróbica máxima en hombres. En el caso de las mujeres, se debe prestar atención a esta situación, ya que sí se observaron diferencias relativas en cuanto a la $\mathrm{P}_{170}$ en aquellas con IMC $\geq 40 \mathrm{~kg} / \mathrm{m}^{2}$. Atribuimos que las diferencias encontradas para las mujeres con mayor IMC podrían estar determinadas por la composición corporal y la distribución de grasa en la mujer. Esta distribución más femoroglutea podría disminuir la capacidad de generar trabajo del tren inferior en la medida que aumenta el 
IMC a diferencia de los hombres en los cuales la distribución de grasa sigue un patrón más del tipo androide. Sin embargo, consideramos como una limitante para dar explicación a esta interpretación de los resultados no tener registro de composición corporal segmentaria de nuestra muestra.

En conclusión, la determinación de la PAM a través de un test submáximo en cicloergómetro en obesos demostró ser un test seguro y bien tolerado. El grado de obesidad no limitó el rendimiento en hombres, pero si en mujeres de mayor IMC. La evaluación y el entrenamiento en el cicloergómetro debe ser considerado como una alternativa válida, ya que el rendimiento de esta población no se ve menoscabado al no tener que transportar su peso corporal. Esta condición puede ser ventajosa frente a otras formas de testeo o entrenamiento aeróbico en las que el transporte del peso corporal provoca un mayor esfuerzo.

\section{Referencias}

1. Martínez JA. Body weight regulation: causes of obesity. Proc Nutr Soc 2000; 59 (3): 337-45.

2. Prentice AM, Jebb SA. Obesity in Britain: gluttony or sloth? BMJ 1999; 311 (7002): 437-9.

3. Hill JO, Wyatt HR, Reed GW, Peters JC. Obesity and the environment: where do we go from here? Science 2000; 299 (5608): 853-5.

4. Arena R, Cahalin L. Evaluation of Cardiorespiratory Fitness and Respiratory Muscle Function in the Obese Population. Prog Cardiovasc Dis 2014; 56 (4): 457-64.

5. Buffart LM, De Backer IC, Schep G, Vreugdenhil A, Brug J, Chinapaw MJ. Fatigue mediates the relationship between physical fitness and quality of life in cancer survivors. J Sci Med Sport 2013; 16 (2): 99-104.

6. Lerdal A, Celius EH, Gunn P. Prescribed Exercise: A Prospective Study of Health Related Quality of Life and Physical Fitness Among Participants in an Officially Sponsored Municipal Physical Training Program. J Phys Act Health 2013; 10 (7): 1016-23.

7. Quinn A, Doody C, O'Shea D. The effect of a physical activity education programme on physical activity, fitness, quality of life and attitudes to exercise in obese females. J Sci Med Sport 2008; 11(5): 469-72.

8. Ross KM, Milsom VA, Rickel KA, Debraganza N, Gibbons LM, Murawski ME, et al. The contributions of weight loss and increased physical fitness to improvements in health-related quality of life. Eat Behav 2009; 10 (2): 84-8.
9. Smart NA, Murison R. Rate of Change in Physical Fitness and Quality of Life and Depression Following Exercise Training in Patients With Congestive Heart Failure. Congest Heart Fail 2012; 19 (1): 1-5.

10. Sorensen LE, Pekkonen MM, Mannikko KH, Louhevaara VA, Smolander J, Alen MJ. Associations between work ability, health-related quality of life, physical activity and fitness among middle-aged men. Appl Ergon 2008; 39 (6): 786-91.

11. Ortega FB, Lee DC, Katzmarzyk PT, Ruiz JR, Sui X, Church TS, et al. The intriguing metabolically healthy but obese phenotype: cardiovascular prognosis and role of fitness. Eur Hear J 2013; 34 (5): 389-97.

12. Gore CJ, Booth ML, Bauman A, Owen N. Utility of pwc75\% as an estimate of aerobic power in epidemiological and population-based studies. Med Sci Sports Exerc 1999; 31 (2): 348-51.

13. Maniscalco M, Arciello A, Zedda A, Faraone S, Verde $\mathrm{R}$, Giardiello C, et al. Right ventricular performance in severe obesity. Effect of weight loss. Eur J Clin Invest 2007; 37 (4): 270-5.

14. Oeser A, Chung CP, Asanuma Y, Avalos I, Stein, CM. Obesity is an independent contributor to functional capacity and inflammation in systemic lupus erythematosus. Arthritis Rheum 2005; 52 (11): 3651-9.

15. Davies B, Daggett A. Responses of adult women to programmed exercise. Br J Sports Med 1977; 11 (3):122-6.

16. Sánchez E, Terreros JL. Valoración de un programa de actividad física informatizado, sobre deportistas de tiempo libre. Archivos Sociedad Chilena de Medicina del Deporte 1992; 9 (35): 243-51.

17. Torok K, Szelenyi Z, Porszasz J, Molnar D. Low physical performance in obese adolescent boys with metabolic syndrome. Int J Obes Relat Metab Disord 2001; 25 (7): 966-70.

18. López Chicharro J, Fernández A. Fisiología del Ejercicio. 3 th Ed. Buenos Aires: Editorial Médica Panamericana. 2008.

19. Astrand PO, Ryhming I. A nomogram for calculation of aerobic capacity (physical fitness) from pulse rate during sub-maximal work. J Appl Physiol 1954; 7 (2): 218-21.

20. Finger J, Krug S, Göbwald A, Hartel S, Bös K. Cardiorespiratory fitness among adults in Germany: Results of the German Helath Interview and Examination Survey for Adults (DEGS1). Bundesgesundheitsblatt 2013; 56: 772-8.

21. Bland J, Pfeiffer K, Eisenmann JC. The PWC170: comparison of different stage lengths in 11-16 year olds. Eur J Appl Physiol 2012; 112 (5): 1955-61.

22. Byrd R, Centry R, Boatwright, D. Effect of inter-repetition rest intervals in circuit weight training on PWC170 
during arm-cranking exercise. J Sports Med Phys Fitness 1988; 28 (4): 336-40.

23. Ho MS, Zander E, Taniges B, Jutzi E, Schulz B. Ergometric heart rate, blood pressure and work capacity (PWC170) in type I diabetics with diabetes-specific microangiopathy. Exp Clin Endocrinol 1983; 82 (3): 291-03.

24. Storer T, Davis J, Caiozzo V. Accurate prediction of $\mathrm{VO}_{2 \max }$ in cycle ergometry. Med Sci Sports Exerc 1990; 22 (5): 704-12.

25. American College of Sports Medicine. ACSM's guidelines for exercise testing and prescription. (7th ed.). Philadelphia, PA: Lippincott Williams and Wilkins, 2006. pp. 106.

26. Borg G, Linderholm H. Perceived Exertion and Pulse Rate during Graded Exercise in Various Age Groups. Acta Medica Scandinavica 1967; 181: 194-206.

27. Duque I, Parra JH, Duvallet A. Maximal aerobic power in patients with chronic low back pain: a comparision with healthy subjects. Eur Spine J 2011; 20 (1): 87-93.

28. Coyle EF, Feltner ME, Kautz SA, Hamilton MT, Montain SJ, Baylor AM, et al. Physiological and biomechanical factors associated with elite endurance cycling performance. Med Sci Sports Exerc 1991; 23 (1): 93-07.

29. Bouchard DR, Langlois MF, Brochu M, Dionne IJ, Baillargeon JP. Metabolically healthy obese women and functional capacity. Metab Syndr Relat Disord 2011; 9 (3): 225-29.

30. De Souza SA, Faintuch J, Fabris SM, Nampo FK, Luz C, Fabio TL, et al. Six-minute walk test: functional capacity of severely obese before and after bariatric surgery. Surg Obes Relat Dis 2009; 5 (5): 540-3.

31. Di Thommazo-Luporini L, Jürgensen SP, Castello-Simoes V, Catai AM, Arena R, Borghi-Silva A. Metabolic and clinical comparative analysis of treadmill six-minute walking test and cardiopulmonary exercise testing in obese and eutrophic women. Rev Bras Fisioter 2012; 16 (6): 469-78.

32. Stegen S, Derave W, Calders P, Van Laethem C, Pattyn P. Physical fitness in morbidly obese patients: effect of gastric bypass surgery and exercise training. Obes Surg 2011; 21 (1): 61-70.

33. Sava F, Laviolette L, Bernard S, Breton MJ, Bourbeau J, Maltais F. The impact of obesity on walking and cycling performance and response to pulmonary rehabilitation in COPD. BMC Pulm Med 2010; 10: 55.

34. Clifford PS, Hellsten Y. Vasodilatory mechanisms in contracting skeletal muscle. J Appl Physiol 2004; 97 (1): 393-403.
35. Reed AS, Tschakovsky ME, Minson CT, Halliwill JR, Torp KD, Nauss LA, et al. Skeletal muscle vasodilatation during sympathoexcitation is not neurally mediated in humans. J Physiol 2000; 15 (1): 253-62.

36. Lambert E, Sari CI, Dawood T, Nguyen J, McGrane M, Eikelis $\mathrm{N}$, et al. Sympathetic nervous system activity is associated with obesity-induced subclinical organ damage in young adults. Hypertension 2010; 56 (3): 351-8.

37. Lafortuna C, Maffiulett N, Agosti F, Sartorio A. Gender variations of body composition, muscle strength and power output in morbid obesity. International Journal of Obesity 2005; 29: 833-41.

38. Vandewalle S, Taes Y, Van Helvoirt M, Herregods N, Ernst C, Roef G, et al. Bone Size an Bone Strength are Increased in Obese Male Adolescents. J Clin Endocrinol Metab 2013; 98 (7): 3019-28.

39. Andersen RE. Exercise, an active lifestyle, and obesity: making the exercise prescription work. Phys Sportsmed 1999; 27 (10): 41-50.

40. Carrel AL, Bernhardt DT.Exercise prescription for the prevention of obesity in adolescents. Curr Sports Med Rep 2004; 3 (6): 330-6.

41. Ho SS, Radavelli-Bagatini S, Dhaliwal SS, Hills AP, Pal $\mathrm{S}$. Resistance, aerobic, and combination training on vascular function in overweight and obese adults. J Clin Hypertens 2012; 14 (12): 848-54.

42. Laskowski ER. The role of exercise in the treatment of obesity. PM R. 2012; 4(11), 840-4.

43. Ross R, Hudson R, Day AG, Lam M. Dose-response effects of exercise on abdominal obesity and risk factors for cardiovascular disease in adults: Study rationale, design and methods. Contemp Clin Trials 2012; 34 (1): 155-60.

44. Voulgari C, Pagoni S, Vinik A, Poirier P. Exercise improves cardiac autonomic function in obesity and diabetes. Metabolism 2012; 62 (5): 609-21.

45. Ekkekakis P, Lind E. Exercise does not feel the same when you are overweight: the impact of self-selected and imposed intensity on affect and exertion. Int J Obes 2006; 30 (4): 652-60.

46. Santarpia L, Contaldo F, Pasanisi F. Body composition changes after weight-loss interventions for overweight and obesity. Clinical Nutrition 2012; 32 (2): 157-61.

47. Faintuch J, Souza SA, Valezi AC, Sant'Anna AF, Gama-Rodrigues JJ. Pulmonary function and aerobic capacity in asymptomatic bariatric candidates with very severe morbid obesity. Rev Hosp Clin Fac Med Sao Paulo 2004; 59 (4): 181-6. 